\title{
Development of an improved competitive ELISA based on a monoclonal antibody against lipopolysaccharide for the detection of bovine brucellosis
}

\author{
Xiaolei Wang ${ }^{1}$, Yan Wang ${ }^{2,3}$, Limei Ma ${ }^{1}$, Ran Zhang ${ }^{1}$, Yanyan De ${ }^{1}$, Xiaowen Yang ${ }^{1}$, Chuanqing Wang ${ }^{2 *}$
} and Qingmin $W^{1 *}$

\begin{abstract}
Background: Brucellosis is the most common bacterial zoonosis, and serological tests are routinely used in brucellosis control and eradication programs. In order to improve the accuracy of serological diagnostic method used in bovine brucellosis detection, this study developed an improved competitive ELISA with higher specificity and good sensitivity.

Results: This study prepared 12 monoclonal antibodies against smooth Brucella lipopolysaccharide. One monoclonal antibody $3 \mathrm{~F}$, presented C epitope specificity, was used to develop a competitive ELISA for the serological detection of bovine brucellosis. The competitive ELISA, a commercial competitive ELISA kit, the rose-bengal plate agglutination test, and a microplate agglutination test were all used in the detection of 6 hyperimmune antisera against other commonly cross-reacted bacterial pathogens and 110 clinical bovine serum samples. The results of the test comparisons indicated that the competitive ELISA had higher specificity than the commercial competitive ELISA kit and RBT, and comparable sensitivity with the commercial ELISA kit.

Conclusions: This study provided a valuable detection tool with high specificity and good sensitivity, which prevent the wrong-culling of bovines in the eradication campaigns of bovine brucellosis.
\end{abstract}

Keywords: Brucellosis, Lipopolysaccharide, Monoclonal antibody, cELISA, Specificity

\section{Background}

Brucellosis is the most common bacterial zoonosis caused by members of the Brucella genus, which infect a wide range of mammals, including dogs, ruminants, humans, and marine mammals. Within the last few years, brucellosis has re-emerged, presenting severe public health challenges and major economic burdens globally [1]. The measures to eradicate and control brucellosis outbreaks are principally based on an intensive testand-slaughter policy $[2,3]$, in which effective technology

\footnotetext{
*Correspondence: wchuanq@163.com; wuqm@cau.edu.cn

${ }^{2}$ Animal Infectious Disease Laboratory, College of Animal Science and Veterinary Medicine, Henan Agricultural University, Zhengzhou, China 'Key Laboratory of Animal Epidemiology and Zoonosis of the Ministry of Agriculture, College of Veterinary Medicine, China Agricultural University, Beijing, China

Full list of author information is available at the end of the article
}

to diagnose brucellosis plays an important role. Although bacterial isolation and identification of Brucella spp. is defined as the 'gold standard' for diagnosis of brucellosis, serological tests are routinely used in brucellosis control and eradication programs. Currently, the common serological diagnosis methods for bovine brucellosis include the serum agglutination test (SAT), the rose-bengal plate agglutination test (RBT), the milk ring test (MRT) $[4,5]$, the complement fixation test (CFT) [6], and primary binding assays such as the indirect ELISA (iELISA) $[7,8]$, the competitive ELISA (cELISA) [9, 10], and the fluorescence polarization assay (FPA) [11]. The majority of serological tests mentioned rely on the detection of antibodies against lipopolysaccharide (LPS). However, false positive results often occur from cross-reaction in the serological detection [12, 13], due to common antigens on LPS of Brucella 
and certain bacteria, especially Yersinia enterocolitica O:9 and Escherichia coli O157 [14, 15]. The sensitivity and specificity of different serological tests are variant [16]. Agglutination tests often do not have very good specificity. The CFT with high specificity and sensitivity has been approved, but tedious operations make it difficult to use for large-scale detection. In the past few decades, the FPA and iELISA with high sensitivity have been used for the diagnosis of brucellosis. The iELISA methods based on LPS antigens easily produce cross-reaction with the antibodies against other bacterial pathogens, which may result in over-culled animals. Unfortunately, the sensitivity of iELISA with protein antigens is not as good as the sensitivity of iELISA utilizing LPS $[17,18]$. The FPA performs excellently for diagnosis but requires expensive specialized apparatus for measurement. These faults indicate that a high-throughput diagnostic methods with good specificity and sensitivity is necessary. The cELISA has become a reliable alternate diagnosis for brucellosis. However, of the limited sensitivity and specificity, the various monoclonal antibodies (MAbs) used in cELISA may result in omission or false detection in practical application. Therefore, an optimal cELISA for the diagnosis of animal brucellosis should be based on the MAb with high specificity and satisfactory properties.

LPS is a major surface antigen of Brucella that can be divided into smooth type (S) or rough type (R) depending on the inclusion or lack of O-polysaccharide (OPS) moiety. Four types of epitopes on the Brucella OPS have been described: the $M$ and $A$ epitopes, present on $M$ and A dominant Brucella strains, respectively; the common (C) epitope, strictly specific for smooth Brucella spp., either A or $\mathrm{M}$ dominant; and the $\mathrm{C} / \mathrm{Y}$ epitope, which is common to smooth Brucella spp. and Y. enterocolitica O:9 [19]. Different OPS epitopes are probably overlapping structures, but the $\mathrm{C}$ epitopes would be important to establish cELISA for the diagnosis of brucellosis.

For the serological detection of Brucella-infected cattle, 12 MAbs against smooth Brucella LPS were produced and characterized. Fortunately, among them, one was identified to be against $\mathrm{C}$ epitope. This MAb was selected to develop a competitive ELISA, which was compared with other methods for the detection of Brucella infection in cattle. The results showed that the developed cELISA demonstrated significantly improved specificity and good sensitivity.

\section{Results}

\section{Screening and characterization of MAb}

This study immunized mice with heat-killed $B$. melitensis $16 \mathrm{M}$ and boosted with large dose of purified LPS (Fig. 1). After four times of cell fusion, hybridomas screened by the iELISA established, twelve positive clones specific to

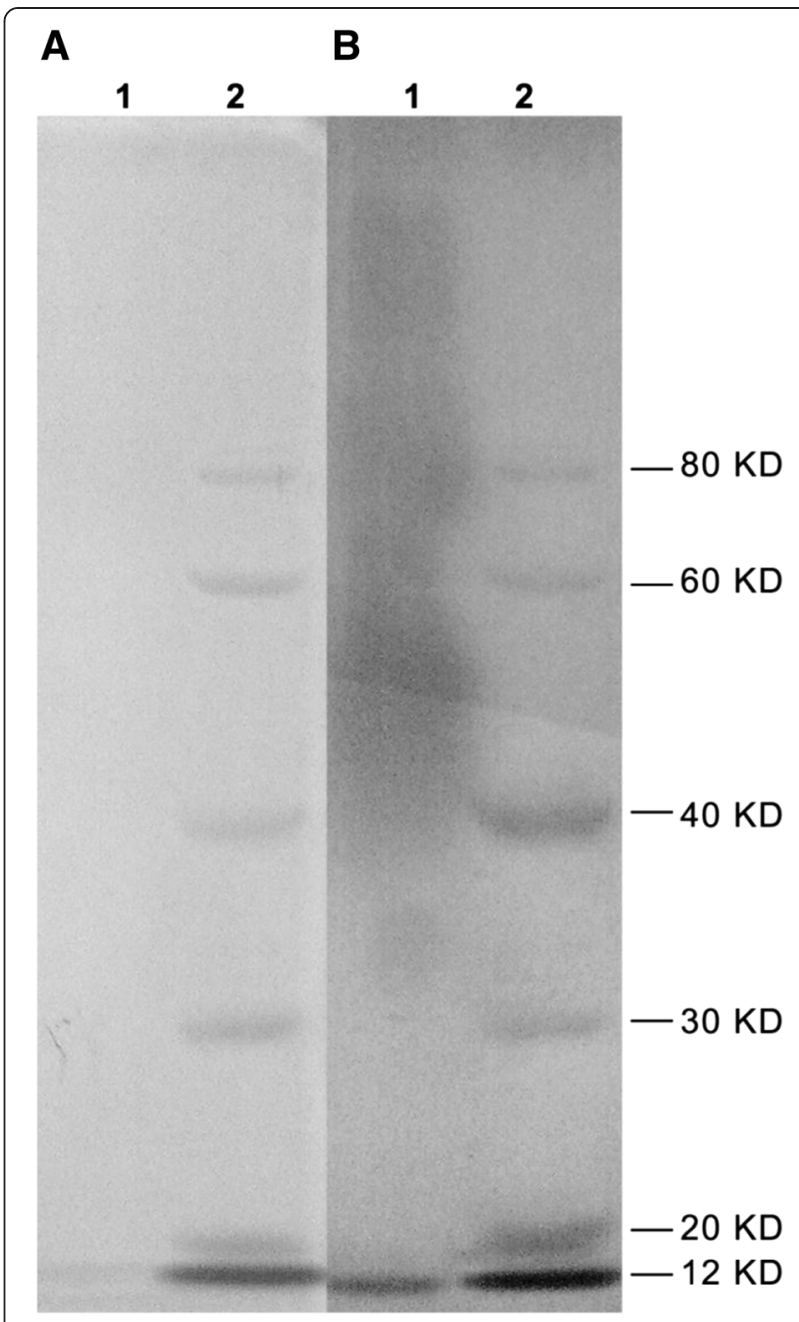

Fig. 1 Coomassie blue-staining (A) and silver-staining (B) of LPS extracted from B.melitensis 16 M. LPS was prepared by hot phenol-water extraction method and fractionated by SDS-PAGE electrophoresis, followed by commassie blue (A) or silver (B) staining. LPS banding is seen (B). The absence of band in commassie blue staining as shown in A indicates no contamination of purified LPS with bacterial proteins. Lane 1: LPS, Lane 2: Molecular weight marker

LPS were obtained and then subcloned three times through the use of a limited dilution method. Among these MAbs, three were IgG1 (II5G1, 6E3, 4C3), four were IgG3 (3H7, $3 \mathrm{~F} 9,2 \mathrm{C} 3$, II4D11), four were IgM (6B8, $6 \mathrm{~F} 2$, $4 \mathrm{H} 7, \mathrm{I} 2 \mathrm{C} 10)$, and one was IgG2a.

\section{Characterization of specificity and epitope of MAb}

In this study, the western blot with whole-cell lysates showed that four MAbs (2C3, 3E3, $3 \mathrm{~F} 9,6 \mathrm{E} 3)$ were specific to B.melitensis $16 \mathrm{M}$. Meanwhile, the other eight MAbs (4H7, 4C3, 6B8, 6 F2, II5G1, II4D11, 3H7, I2C10) recognized epitope $\mathrm{C} / \mathrm{Y}$ because they had weak crossreactivity with $Y$. enterocolitica O:9 (Fig. 2). The results from the ELISA were consistent with the western blot test (Table 1). 


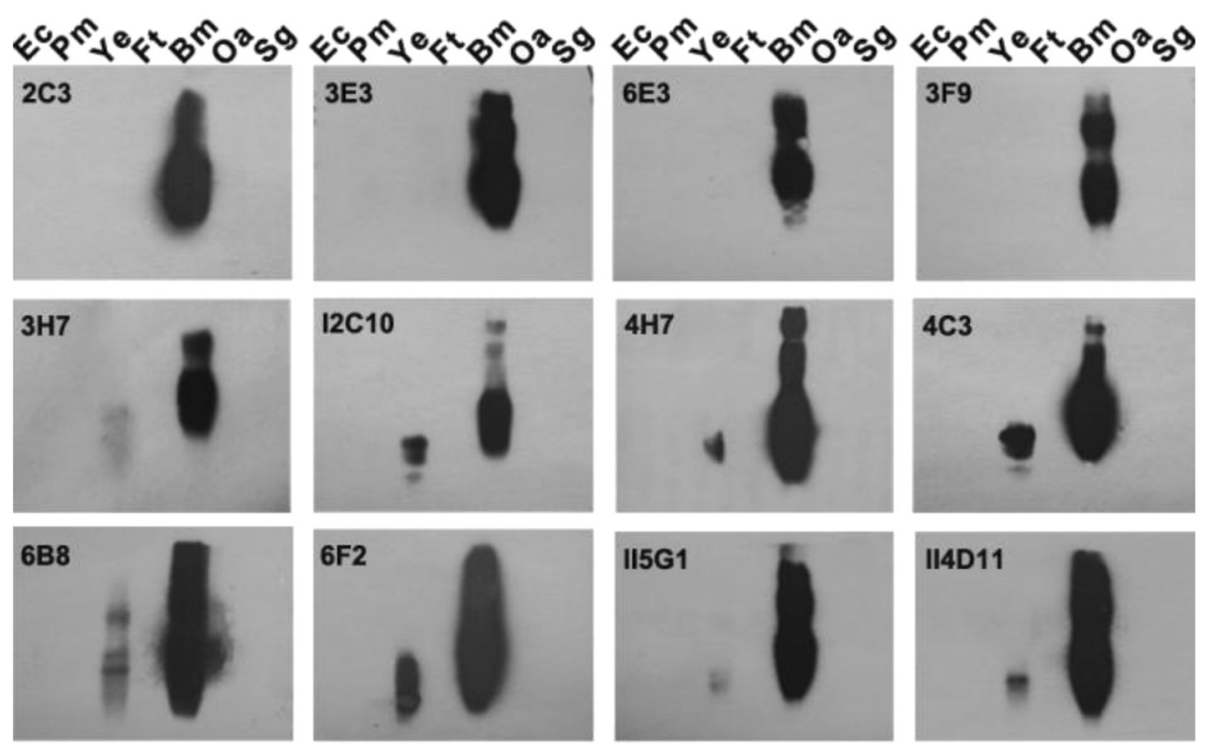

Fig. 2 Western blot analysis for specifity of MAbs. Specificity of MAbs was investigated with whole-cell extracts of Brucella melitensis 16 M and different pathogens. Western blot were carried out as described in Materials and Methods. Abbreviation: Ec: E. coli O157; Pm: P. multocida; Ye: Y. enterocolitica O:9; Ft: F. tularensis LVS; Bm: B. melitensis 16 M; Oa: O. anthropic ATCC49188; Sg: S. gallinarum

$\mathrm{C}$ epitopes have been subdivided and seven epitopes on the Brucella OPS have been defined, including: $A, M$, $\mathrm{C}(\mathrm{M}>\mathrm{A}), \mathrm{C}(\mathrm{A}=\mathrm{M}), \mathrm{C} / \mathrm{Y}(\mathrm{M}>\mathrm{A}), \mathrm{C} / \mathrm{Y}(\mathrm{A}=\mathrm{M})$, and $\mathrm{C} /$ $\mathrm{Y}(\mathrm{A}>\mathrm{M})[20,21]$. Further analysis of the MAb epitope specificity was performed with native rough phenotype Brucella and with smooth Brucella strains of three serotypes, i.e., $\mathrm{A}^{+} \mathrm{M}^{-}, \mathrm{A}^{-} \mathrm{M}^{+}$, and $\mathrm{A}^{+} \mathrm{M}^{+}$, corresponding to strains expressing mainly the $\mathrm{A}$ (A-dominant) or $\mathrm{M}$ (M-dominant) antigen or both antigens in nearly equivalent amounts [22] (Fig. 3). All the MAbs expect MAb
6B8, had no reactivity with rough type B. canis RM 6/66. $\mathrm{MAb} 2 \mathrm{C} 3$, 3E3, 6E3 reacted only with $B$. melitensis $16 \mathrm{M}$ ((M-dominant, $\left.\mathrm{A}^{-} \mathrm{M}^{+}\right)$, which confirmed the specificity for $M$ epitope. MAb 3 F9 was thought to be specific for the $C$ epitope, as it bound to $B$. melitensis $16 \mathrm{M}, B$. abortus 2308 (A-dominant, $\mathrm{A}^{+} \mathrm{M}^{-}$), and $B$. melitensis $\mathrm{NI}\left(\mathrm{A}^{+} \mathrm{M}^{+}\right)$.

iELISA was performed to measure the MAbs relative binding level (Table 1). MAb 3 F9 bound equally to $B$. melitensis $16 \mathrm{M}$ and B. melitensis $\mathrm{NI}$, but had a weaker

Table 1 Indirect ELISA results for hybridoma supernatants

\begin{tabular}{|c|c|c|c|c|c|c|c|c|c|c|c|c|}
\hline \multirow[t]{2}{*}{ MAb } & \multirow{2}{*}{$\begin{array}{l}\text { lg } \\
\text { class }\end{array}$} & \multicolumn{10}{|l|}{$O D^{a}$} & \multirow{2}{*}{$\begin{array}{l}\text { Epitope } \\
\text {-recognized }\end{array}$} \\
\hline & & Y. enterocolitica $0: 9$ & $\begin{array}{l}\text { E. coli } \\
\text { O157 }\end{array}$ & S. gallinarum & O.anthropi & F.tularensis & P.multocida & $R M 6 / 66(\mathrm{R})$ & $\begin{array}{l}\mathrm{Nl} \\
\left(\mathrm{A}^{+} \mathrm{M}^{+}\right)\end{array}$ & $\begin{array}{l}16 \mathrm{M} \\
\left(\mathrm{A}^{-} \mathrm{M}^{+}\right)\end{array}$ & $\begin{array}{l}2308 \\
\left(A^{+} M^{-}\right)\end{array}$ & \\
\hline $2 \mathrm{C3}$ & $\operatorname{lgG} 3$ & 0.043 & 0.038 & 0.027 & 0.035 & 0.030 & 0.033 & 0.044 & 1.176 & 0.856 & 0.040 & M \\
\hline $3 \mathrm{E} 3$ & $\lg G 1$ & 0.037 & 0.053 & 0.036 & 0.039 & 0.031 & 0.042 & 0.041 & 1.419 & 0.942 & 0.044 & M \\
\hline $6 \mathrm{E} 3$ & $\lg G 1$ & 0.040 & 0.026 & 0.023 & 0.028 & 0.025 & 0.024 & 0.031 & 1.086 & 0.907 & 0.060 & M \\
\hline $3 \mathrm{H} 7$ & $\lg G 3$ & 0.350 & 0.061 & 0.046 & 0.045 & 0.043 & 0.057 & 0.035 & 1.115 & 0.567 & 1.544 & $C / Y(A>M)$ \\
\hline 3 F9 & $\lg G 3$ & 0.037 & 0.053 & 0.031 & 0.042 & 0.037 & 0.049 & 0.034 & 1.022 & 0.983 & 0.514 & $C(M>A)$ \\
\hline $4 C 3$ & $\lg G 2 a$ & 0.422 & 0.031 & 0.028 & 0.033 & 0.027 & 0.030 & 0.043 & 0.647 & 0.687 & 0.605 & $C / Y(M=A)$ \\
\hline $115 \mathrm{G} 1$ & $\lg G 1$ & 0.434 & 0.063 & 0.038 & 0.048 & 0.041 & 0.045 & 0.034 & 0.906 & 0.882 & 1.113 & $C / Y(M=A)$ \\
\hline ||4D11 & $\lg G 3$ & 0.557 & 0.043 & 0.034 & 0.041 & 0.031 & 0.042 & 0.032 & 0.938 & 0.930 & 0.899 & $C / Y(M=A)$ \\
\hline $6 B 8$ & $\lg M$ & 1.039 & 0.037 & 0.030 & 0.036 & 0.032 & 0.039 & 0.205 & 0.837 & 0.913 & 0.991 & $C / Y(M=A)$ \\
\hline $6 F 2$ & $\lg M$ & 0.959 & 0.023 & 0.021 & 0.033 & 0.031 & 0.024 & 0.048 & 0.897 & 0.899 & 0.845 & $C / Y(M=A)$ \\
\hline $4 \mathrm{H} 7$ & $\lg M$ & 0.442 & 0.030 & 0.028 & 0.035 & 0.030 & 0.034 & 0.043 & 0.946 & 0.853 & 0.898 & $C / Y(M=A)$ \\
\hline 12C10 & $\lg M$ & 0.503 & 0.058 & 0.033 & 0.040 & 0.028 & 0.044 & 0.040 & 0.896 & 0.689 & 1.317 & $C / Y(A>M)$ \\
\hline
\end{tabular}

${ }^{a} \mathrm{OD}$ of hybridoma supernatants in dilutions of OD 1.0 in iELISA with LPS of B.melitensis $16 \mathrm{M}$ 

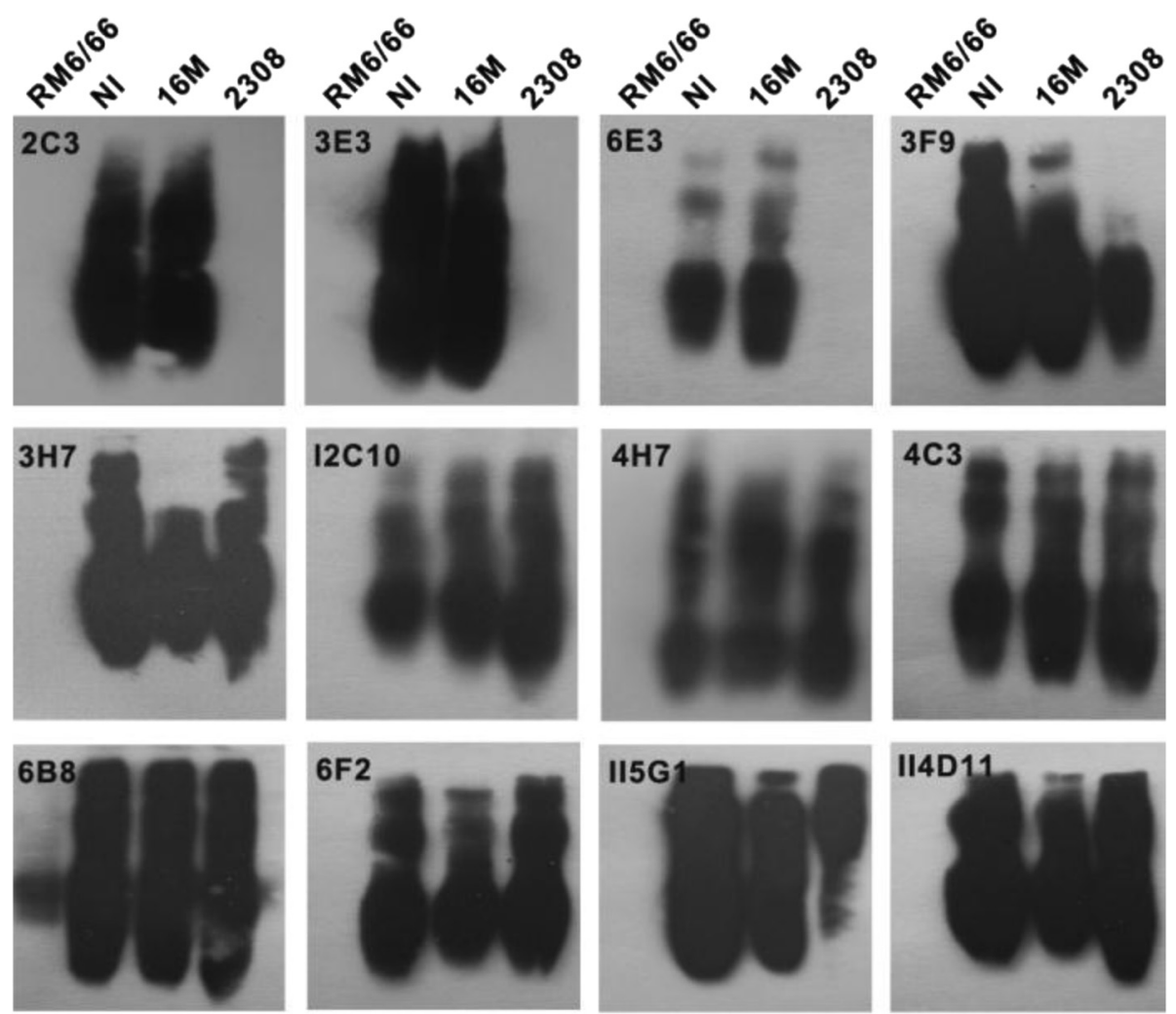

Fig. 3 Western blot analysis for epitope of MAbs. Whole-cell extracts of B. canis RM6/66 (native rough type), B. melitensis $\mathrm{NI}$ (smooth type, $A^{+} M^{+}$), B. melitensis $16 \mathrm{M}$ (smooth type, M-dominant, $\mathrm{A}^{-} \mathrm{M}^{+}$), B. abortus 2308 (smooth type, $A$-dominant, $\mathrm{A}^{+} \mathrm{M}^{-}$) were used to analyze the epitopic specificity of MAbs. SDS-PAGE and Western blot were carried out as described in Materials and Methods

bind to B. abortus 2308, and thus, it recognized $\mathrm{C}(\mathrm{M}>\mathrm{A})$ epitope. Aside from binding to $Y$. enterocolitica $\mathrm{O}: 9$, the binding level of MAbs 4H7, 4C3, 6B8, 6 F2, II5G1, II4D11 were generally equal relative to $\mathrm{A}$ or $\mathrm{M}$ dominance. These results confirmed their $\mathrm{C} / \mathrm{Y}(\mathrm{A}=\mathrm{M})$ specificity. As $\mathrm{MAb}$ $3 \mathrm{H} 7, \mathrm{I} 2 \mathrm{C} 10$ bound to the A-dominant strain significantly stronger than to the M-dominant strain, they were thought to be specific for the $\mathrm{C} / \mathrm{Y}(\mathrm{A}>\mathrm{M})$ epitope.

\section{Determination of cut-off value and specificity of cELISA}

On account of its specificity for C epitope, MAb 3 F9 was selected to develop a cELISA. After protocol optimization of blocking solution, dilution ratio of MAb and sample respectively, 63 negative bovine serum samples were used to determine the cut-off value. As the $\mathrm{p}$-value of negative sera PI analyzed with the Shapiro-Wilk test was 0.482 , this paper concluded that the data were distributed normally. The mean PI value was $22 \%(\mathrm{SD}=9 \%)$ for the bovine. Therefore, the cut-off value (mean $+2 \mathrm{SD})$ to determine the status of the serum samples in response to smooth Brucella was set at $40 \%$ (Fig. 4A).

To confirm its specificity, the cELISA was used to detect the polyclonal antisera against E. coli O157, S. gallinarum, P. multocida, $Y$. enterocolitica O:9, F. tularensis LVS, and O. anthropic ATCC49188. The results showed that the PI values of all of these sera were much lower than the cut-off value (Fig. 4B).

\section{Comparison among cELISA,a commercial cELISA kit, RBT and MAT}

Six hyperimmune antisera and 110 clinical bovine serum samples were tested with the cELISA. The resulting data were compared with data obtained from a commercial cELISA kit, the RBT and the MAT. Five of the six hyperimmune antisera were negative in the four diagnostic tests. Notably, the antisera of $Y$. enterocolitica O:9 detected as negative in the cELISA and the MAT, but as positive in the commercial kit and the RBT (Table 2).

Among the bovine sera, the positive rate detected by the cELISA was $54.5 \%(60 / 110)$, compared to $48.2 \%$ (53/110), $62.7 \%(69 / 110)$, and $42.7 \%(47 / 110)$ by the commercial kit, RBT, and MAT, respectively. Data appear in Table 3, by all four methods, 40 sera were tested negative and 43 sera tested positive. One sample tested positive in the commercial kit but negative in the other three tests. The additional 26 serum samples tested positive with RBT, and of those sera, 17 were 

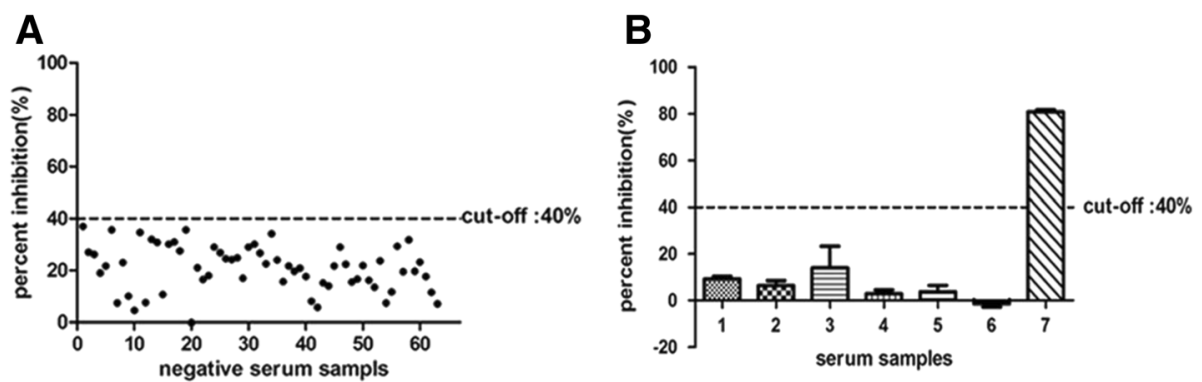

Fig. 4 Establishment of the cELISA. (A) Percent inhibition values of 63 negative serum samples by cELISA. A cut-off value was set at $40 \%$ (mean + 2SD). (B) Percent inhibition of polyclonal antisera against various bacteria serological related with smooth Brucella. 1 Y. enterocolitica O:9; 2 E. coli O157; 3S. gallinarum; 4 O. anthropic ATCC49188; 5 F. tularensis LVS; 6 P. multocida; 7 positive bovine sera. Only the positive bovine sera had a PI exceeding the cut-off value. The Error bars indicate the standard deviations from three-well replications for each serum sample

confirmed positive with the cELISA, while the commercial kit and the MAT missed these positive sera (5 were missed by both the commercial kit and the MAT, 4 by just the commercial kit, and 8 by just the MAT). Eight sera were negative, with the exception of the RBT, and one was negative in the cELISA and the MAT, but positive in the commercial kit and RBT. The cELISA agreed well with the commercial kit $(\mathrm{kappa}=0.801)$, the RBT $($ kappa $=0.832)$, and the MAT (kappa $=0.767)$. The supplement shows detail detection results of all of the serum samples (Additional file 1: Table S1).

\section{Discussion}

Domestic animals infected with Brucella spp. are culled partly based on the detection of the antibodies against Brucella LPS antigen. However, other bacterial infections often disturb the serological examination of brucellosis [12, 13], due to the antigens of cross-reaction among the bacteria. It is known that smooth Brucella LPS contains O-polysaccharide (OPS) moiety, which has been divided into seven epitopic specificities: $A, M, C(M>A)$, $\mathrm{C}(\mathrm{A}=\mathrm{M}), \mathrm{C} / \mathrm{Y}(\mathrm{M}>\mathrm{A}), \mathrm{C} / \mathrm{Y}(\mathrm{A}=\mathrm{M})$, and $\mathrm{C} / \mathrm{Y}(\mathrm{A}>\mathrm{M})$ $[20,21]$. Research has shown that the cross-reactions in the brucellosis serological detection mainly occur as a result of the similar structures that $A$ and $C / Y$ epitopes share with the OPS of $Y$. enterocolitica O:9 and other bacteria $[14,15]$. These possible inaccuracies in serological

Table 2 Results of 6 hyperimmune antisera detection by cELISA, commercial kit, RBT and MAT

\begin{tabular}{lllll}
\hline Sample name & CELISA & Commercial kit & RBT & MAT \\
\hline E. coli O157 & - & - & - & - \\
O. anthropic & - & - & - & - \\
F. tularensis LVS & - & - & - & - \\
S. gallinarum & - & - & - & - \\
Y. enterocolitica O:9 & - & + & + & - \\
P. multocida & - & - & - & -
\end{tabular}

$-:$ negative result, +: positive result examination methods due to cross-reaction may result in the wrong-culling of animals in the campaigns to eradicate animal brucellosis.

In this study, 12 MAbs against LPS of Brucella melitensis $16 \mathrm{M}$ were characterized, among which four were IgM MAbs, four were IgG3, three were IgG1, and one was IgG2a. Brucella strains of three serotypes belonging to Brucella melitensis and Brucella abortus were used as representations to identify the epitope specificity of MAbs. Brucella suis, which possess five biovars of three serotypes were not used due to the lack of the strains in the laboratory. As Brucella suis biovar 2 displayed unique reactivity with MAbs of $C(A=M)$ and $C(M>A)$ [21], identification of MAbs with Brucella suis biovar 2 may provide some interesting results. Almost all of the Mabs were against the $\mathrm{C} / \mathrm{Y}$ or $\mathrm{M}$ epitopes, with the exception of MAb 3 F9. Interestingly, among the six MAbs that recognized $\mathrm{C} / \mathrm{Y}(\mathrm{A}=\mathrm{M})$ epitope, MAb 6B8 reacted faintly with B. canis RM 6/66 (Table 1, Fig. 3), which led this study to analyze whether there were discrepancies between the epitopes of these MAbs. The ELISA additivity test [23] was applied to the six MAbs, and the results (see Additional file 1) showed that either two MAbs failed to bind simultaneously to the antigen. As the core oligosaccharide is common to rough and smooth LPS, MAb 6B8 likely differed from the others by recognizing this region rather than just the formamido-mannose polymer in OPS [24]. The epitope of MAb 6B8 necessitates further analysis.

Fortunately, one monoclonal antibody 3 F9, which belonged to the IgG3 isotype, was identified to be specific for $\mathrm{C}$ epitope as evidenced by western blot and iELISA in this study. MAb 3 F9 indicates good specificity, as it did not cross-react with E. coli O157, S. gallinarum, P. multocida, Y. enterocolitica O:9, F. tularensis LVS, or O. anthropic ATCC49188. Furthermore, the MAb 3 F9 was used to establish a successful cELISA for the examination of bovine brucellosis. As Cattle is mainly infected by $B$. abortus, sometimes can also be infected by B.melitensis 
Table 3 Results of 110 bovine sera samples for Brucella antibody detection by cELISA, commercial kit, RBT and MAT

\begin{tabular}{lllll}
\hline Sample number & CELISA & commercial kit & RBT & MAT \\
\hline 43 & + & + & + & + \\
40 & - & - & - & - \\
8 & + & + & + & - \\
8 & - & - & + & - \\
5 & + & - & + & - \\
4 & + & - & + & + \\
1 & - & + & + & - \\
1 & - & + & - & - \\
\hline
\end{tabular}

-: negative result, + : positive result

and by $B$. suis when they share pastures or facilities with infected pigs, goats and sheep $[25,26]$, the method in this study would be extensively applicable because Brucella C epitopes appear in all smooth Brucella spp. Besides, this proposed method could eliminate the nonspecific serological reaction in the examination of bovine brucellosis.

The established cELISA was compared with RBT, MAT and a commercial cELISA kit for the detection of six hyperimmune antisera and 110 clinical bovine serum samples. The results showed that all of six hyperimmune antisera, including antisera against E. coli O157 and $Y$. enterocolitica O:9 that commonly cross-reacted in brucellosis serological diagnosis, presented negative responses in the cELISA test. However, the hyperimmune antisera against $Y$. enterocolitica O:9 tested positive in the commercial kit and RBT. MAT test determined its antibody titer to be 25 . The results demonstrate that the cELISA established in this study possesses higher specificity than the commercial cELISA and RBT. Multiple serum samples from animals infected with these pathogens may be more suitable for evaluation of the specificity. However, in consideration of the hyperimmune antisera used in this test with higher titer and affinity than clinical sera, it's persuasive for the good specificity of the cELISA.

The results of 110 clinical bovine sarum samples by the cELISA coincided with the results of the commercial kit and RBT at rates of $90 \%$ (99/110), and $91.82 \%$ (101/ $110)$, respectively. All the samples appearing negative in RBT test also tested negative in the improved cELISA. Moreover, the samples that had antibody titers over 200 in MAT test also had a positive response in the cELISA, the commercial kit, and RBT tests. These results shown in detail in an additional table (see Additional file 2) suggest that the cELISA has a good detection performance. Of samples positive in RBT test and of the antibody titers 50-100 in MAT test, $100 \%(23 / 23)$ samples tested positive in the cELISA test, and only $69.6 \%(16 / 23)$ tested positive in the commercial cELISA test. These results indicate that the cELISA may have better sensitivity in the detection of serological positive state with low antibody titer.
A further accurate comparison may need more serum samples in status of low antibody titer. These comparative results also showed that RBT was the most sensitive and suitable for screening test in bovine brucellosis.

Although this preliminary results indicated the applicable prospects of the cELISA for the specific detection of bovine brucellosis, further evaluating and optimizing the detection performance of this method was indispensable in the future with the standardized positive and negative sera, and more clinical bovine serum samples. It was reported that cELISA could be used to distinguish vaccinated antibody $[9,16]$. In this study the ability of differentiating cattle vaccinated with S19 from infected with Brucella field strains was also required for evaluation of the detection performance of the cELISA. Some Brucella-infected animals reacted negatively to serological tests [27, 28], which indicated an early-stage infection or the bacterial loads were too few. More research should be conducted on these cases.

\section{Conclusions}

This study prepared twelve monoclonal antibodies against smooth Brucella lipopolysaccharides through cell fusions and then characterized them by western blot and iELISA. One monoclonal antibody 3 F9 presented the IgG3 subclass, C epitope specificity. Mab 3 F9 was used to establish a cELISA for the detection of bovine brucellosis. A comparison of the cELISA with a commercial cELISA kit, RBT and MAT showed that, the cELISA had more specificity than the commercial cELISA kit and RBT, and comparable sensitivity with the commercial cELISA kit. This study provided a valuable detection tool with higher specificity and good sensitivity, which prevent the wrong-culling of bovines in the eradication campaigns of bovine brucellosis.

\section{Methods \\ Bacterial strains}

The B. abortus 2308, B. melitensis $16 \mathrm{M}$, and B. canis RM6/66 were all kindly donated by Qianni He (Institute of Veterinary Research, Xinjiang Academy of Animal Sciences, China). These strains were originally collected and preserved in the China Veterinary Culture Collection Center (CVCC, Beijing, China). The epidemic strain $B$. melitensis NI was isolated from an aborted bovine fetus from Inner Mongolia by this laboratory. Escherichia coli O157 and Salmonella gallinarum were preserved in the laboratory and originally collected in CVCC. Ochrobactrum anthropic ATCC49188 was purchased from the Guangdong Microbiology Culture Center. The inactivated culture of Yersinia enterocolitica O:9 and Francisella tularensis Live Vaccine Strain were gifts from the Huaiqi Jing (National Institute for Communicable Disease Control and Prevention, Chinese Center for Disease Control and Prevention, Beijing, 
China) and the Jingliang Su (China Agricultural University, Beijing, China) respectively. Pasteurella multocida was isolated from the field and identified in this laboratory previously. Brucella strains and O. anthropic ATCC49188 were routinely grown in either tryptic soy broth (BD, USA) or tryptic soy agar. The E. coli $\mathrm{O} 157$ and the S. gallinarum were grown in Luria-Bertani, and the P. multocida was grown in Brain Heart Infusion Broth (BD, USA). All operations with living Brucella strains were performed in biosafety level 3 facilities at China Agricultural University.

\section{Serum samples}

Negative bovine serum samples were collected from cattle without a history of Brucella infection. Clinical bovine serum samples were collected from the Beijing and Hebei provinece in China and stored at $-20{ }^{\circ} \mathrm{C}$.

Rabbit hyperimmune antisera against E. coli O157, S. gallinarum, $P$. multocida, $Y$. enterocolitica O:9, F. tularensis LVS, and O. anthropic ATCC49188 were all prepared previously with immunization with inactivated cultures four times every two weeks. The antisera were used for the specificity analysis of the established cELISA.

\section{Antigenic preparations}

For mice immunization and MAb screening, LPS was prepared from B. melitensis $16 \mathrm{M}$ by the hot phenolwater method [29] and then analyzed for purity by silver-staining and Coomassie Blue Staining [30]. The Brucella strains were heat-killed at $68{ }^{\circ} \mathrm{C}$ for $2 \mathrm{~h}$, and the other bacteria were inactivated with $0.2 \%$ formaldehyde. For MAb specificity and epitope analysis, all the bacteria were resuspended with PBS and adjusted to $\mathrm{OD}_{600}$ approximately 1.0. For western blot, cell lysates were obtained by boiling in the presence of SDS. For ELISA, whole-cell lysates were obtained by ultrasonication.

\section{Establishment of MAb cell lines}

Five 4 to 6-week-old female BALB/c mice were purchased from the Jinmuyang Laboratory Animal Breeding Co., LTD (Beijing, China). All animal studies complied with the guidelines for laboratory animal welfare and ethics set forth by the Beijing Administration Committee of Laboratory Animals and were approved by the Animal Care and Use Committee of China Agricultural University. The mice were immunized with $10^{8} \mathrm{CFU}$ heatkilled B. melitensis $16 \mathrm{M}$ organisms by intraperitoneal injection. The mice were boosted with the same dose at Week 3 and Week 5. Blood was collected from the tail in order to check the antibody titer, and then the mouse producing the highest titer was selected for hybridoma production. Serial boosters using an intraperitoneal injection of $50 \mu \mathrm{g}$ of $B$. melitensis $16 \mathrm{M}$ LPS were given every day during the three days prior to the cell fusion to improve the positive rate of antibody secreting hybridoma. Spleen cells from immunized mice were fused with $\mathrm{Sp} 2 / 0$ mouse myeloma cells at a ratio of 5:1 in the presence of $50 \%(\mathrm{w} / \mathrm{v})$ PEG 4000. Then, the fused cells were plated into 96-well microplates in DMEM with HAT media supplemented with $20 \%$ fetal bovine serum. The hybridoma cells were cultured in the HAT media for the first 7 days and then in the HT media for the next 7 days. The culture supernatants were screened for specific antibodies against LPS by iELISA as described below. The positive hybridoma clones were subcloned three times by limiting dilution until monoclones were obtained. The stable antibody-producing clones were expanded and cryopreserved in liquid nitrogen. MAb culture supernatants were produced and collected as described previously [31].

\section{iELISA for screening MAb}

The 96-well microplates were coated with LPS $(0.5 \mu \mathrm{g} /$ $\mathrm{ml}, 100 \mu \mathrm{l} /$ well in $0.05 \mathrm{M}$ carbonate buffer, $\mathrm{pH}$ 9.6) at $4^{\circ}$ $\mathrm{C}$ overnight. Nonspecific protein binding was blocked with $5 \%$ skim milk in PBST (0.01 M, pH7.2 PBS containing $0.05 \%$ Tween-20) at $37{ }^{\circ} \mathrm{C}$ for $30 \mathrm{~min}$. Hybridoma supernatants and HRP-conjugated goat antimouse IgG (KPL, USA) diluted 1:5,000 in PBST were sequentially added and incubated at $37{ }^{\circ} \mathrm{C}$ for $1 \mathrm{~h}$. Substrate solution containing $3,3^{\prime}, 5,5^{\prime}$-tetramethylbenzidine (TMB) was added to each well at $37^{\circ} \mathrm{C}$ for $10 \mathrm{~min}$ for visualization. The reaction was stopped with $2 \mathrm{M}$ sulfuric acid. An automatic ELISA plate reader (BioTek synergy ${ }^{\mathrm{n}} 2$, USA) was used to measure the absorbance at $450 \mathrm{~nm}$, and OD above 0.1 was consider positive.

\section{ELISA to determine the isotype of MAb}

The isotype of the MAbs were determined by testing the hybridoma cell culture supernatants with a mouse monoclonal antibody isotyping kit (SBA, USA), according to the manufacturer's instructions.

\section{Analysis for MAb specificity and epitope}

In Western blot, whole-cell extracts of Brucella and other bacteria were resolved by $12 \%$ SDS-PAGE and then transferred to a nitrocellulose membrane. The membrane was blocked with $10 \%$ skim milk at room temperature for $3 \mathrm{~h}$, then incubated with MAb supernatants at room temperature for $1 \mathrm{~h}$. Unbounded antibody was washed off, and then the HRP-conjugated goat anti-mouse IgG was incubated on the membrane at room temperature for $50 \mathrm{~min}$. Unbound conjugate was then washed off, and the membrane was added to ECL substrate, placed under an $\mathrm{x}$-ray film, and exposed in a dark room.

In iELISA, 96-well microplates were incubated at room temperature overnight with whole-cell lysates $100 \mu \mathrm{l}$ per well. After blocking, the MAb supernatants 
were added to each well, and the ELISA was conducted as described above.

\section{Establishment of cELISA}

The microplates were coated with heat-killed wholecell B. melitensis NI cultures in PBS $\left(\mathrm{OD}_{600}\right.$ approximately 1.0) $10 \mu \mathrm{l} /$ well, and then placed in an incubator at $40-50{ }^{\circ} \mathrm{C}$ until the liquid completely evaporated. Next, $5 \%$ glutaraldehyde solution in $0.1 \mathrm{M}$ sodium bicarbonate was added to fix the antigens and left at room temperature overnight. After blocking with $5 \%$ skim milk at $37{ }^{\circ} \mathrm{C}$ for $1 \mathrm{~h}, 50 \mu \mathrm{l}$ of the serum sample 1:20 diluted in blocking agents was added to each well, followed immediately by the addition of $50 \mu \mathrm{l}$ of MAb 3 F9 supernatants 1:400 diluted in blocking agents. The plates were incubated at $37{ }^{\circ} \mathrm{C}$ for $1 \mathrm{~h}$ with shaking during the initial $3 \mathrm{~min}$. Set up in duplicate wells, the controls included a strong positive, a weak positive, a negative control serum, and a buffer control. After washing with PBST, HRPconjugated goat anti-mouse IgG 1:6000 diluted in PBST was added and incubated at $37{ }^{\circ} \mathrm{C}$ for $40 \mathrm{~min}$. The unbound conjugates were removed by washing, and the chromogenic reaction was achieved as described above. As the bottoms of the plates were covered by the glutaraldehyde solution, two wavelength $(450 \mathrm{~nm}$ and $630 \mathrm{~nm}$ ) were used to measure the OD, i.e. $\mathrm{OD}_{450}-\mathrm{OD}_{630}$. The percent inhibition (PI) value was determined using the formula: PI (\%) $=100-(\mathrm{OD}$ [test sample] / OD [buffer control] $\times 100$ ).

Sixty-three bovine serum samples from cattle without a history of Brucella infection, which were previously confirmed negative through the RBT and a microplate agglutination test (MAT), were used to determine the PI cut-off value that was designed as the mean PI of negative sera +2 standard deviations (SD), in order to ensure that $95 \%$ of PI values for the negative sera fell within this range.

To evaluate its specificity, the cELISA was used to detect polyclonal antisera against E. coli O157, S. gallinarum, $P$. multocida, $Y$. enterocolitica O:9, F. tularensis LVS, and O. anthropic ATCC49188. PI for these antisera were also calculated.

\section{Comparisons of cELISA, a commercial cELISA kit, RBT and MAT}

The 6 polyclonal antisera mentioned above and 110 bovine serum samples were diagnosed using the cELISA, a commercial cELISA kit (SVANOVIR ${ }^{\circ} \mathrm{Bru}^{-}$ cella-Ab C-ELISA, Sweden), RBT, and MAT. The ELISA kit was used in the manner as recommended by the manufacturer. The RBT was operated as previously described [22]. The MAT was performed as reported [32], but with some modification. Serum samples diluted 1:12.5 and 2-folded serially in the PBS were prepared in a 96-well V-bottom microplate. An equal volume $(50 \mu \mathrm{L})$ of antigen solution was added to each well. The sealed plates were incubated in a humid atmosphere at $37{ }^{\circ} \mathrm{C}$ for $24 \mathrm{~h}$. The titer of serum samples was expressed as reciprocal of the highest dilution of sera showing completely agglutination. The positive serum samples had titer 100 or more, and the suspicious serum samples had titer of 50. The smooth antigens used in the RBT and the MAT were purchased from the China Institute of Veterinary Drug Control.

\section{Statistical analysis}

SPSS v20.0 software was used to analyze the result of a normality test of the negative sera PI values using a Shapiro-Wilk test. The software was also used to analyze the degree of agreement between the cELISA, a commercial kit, RBT, and MAT by kappa statistics.

\section{Additional files}

Additional file 1: Epitope mapping of MAbs $(C / Y(M=A))$ on additivity test. The additivity index (Al) value were calculated. The value below 50 indicated that the MAbs tested recognized the same epitope.

Additional file 2: Detection results of four serological diagnosis.

The detail detection results of 110 bovine sera samples and 6 hyperimmune antisera in four serological diagnosis were shown in the table.

\section{Abbreviations}

MAb: monoclonal antibody; RBT: the rose-bengal plate agglutination test; tCFT: he complement fixation test; FPA: the fluorescence polarization assay; MAT: microplate agglutination test; CELISA: competitive ELISA; iELISA: indirect ELISA; LPS: lipopolysaccharide; PI: percent inhibition.

\section{Competing interests}

The authors declare that they have no competing interests.

\section{Authors' contributions}

XLW and QMW took the lead on the the design of the study. XLW, YW and RZ produced and characterized the monoclonal antibodies. XLW and LMM performed the optimization of the CELISA procedure. LMM, YYD and XWY carried out the comparison of four diagnostic tests. CQW provided critical feedback on the manuscript. XLW and QMW wrote the manuscript. All authors read and approved the final manuscript.

\section{Acknowledgments}

We acknowledged Qianni He, Huaiqi Jing and Jingliang Su for the providing of strains. We also acknowledged the assistance of Leying Zhang, Ning Wang, Mengmeng Hu, Chongyan Xu for collecting the clinical bovine sera. This work was supported by the National Natural Science Foundation of China (31372446), and the Special Fund for Agro-Scientific Research in the Public Interest (200903027).

\section{Author details}

${ }^{1}$ Key Laboratory of Animal Epidemiology and Zoonosis of the Ministry of Agriculture, College of Veterinary Medicine, China Agricultural University, Beijing, China. ${ }^{2}$ Animal Infectious Disease Laboratory, College of Animal Science and Veterinary Medicine, Henan Agricultural University, Zhengzhou, China. ${ }^{3}$ Zhumadian Animal Disease Control Center, Zhumadian, China.

Received: 10 December 2014 Accepted: 12 May 2015

Published online: 21 May 2015 


\section{References}

1. Seleem MN, Boyle SM, Sriranganathan N. Brucellosis: A re-emerging zoonosis. Vet Microbiol. 2010;140(3-4):392-8.

2. Martins H, Garin-Bastuji B, Lima F, Flor L, Pina Fonseca A, Boinas F. Eradication of bovine brucellosis in the Azores, Portugal-Outcome of a 5-year programme (2002-2007) based on test-and-slaughter and RB51 vaccination. Prev Vet Med 2009;90(1-2):80-9.

3. Park SY, Kim TJ, Yoon H, Kim JY, Lee MJ, Lee WC. A retrospective study of the extensive eradication program for brucellosis outbreaks and control in Korea, 2002-2009. Jpn J Infect Dis. 2012;65(5):427-9.

4. Gurturk K, Boynukara B, Ithan Z, Hakki Ekin I, Gulhan T. Comparison of the dot-immunobinding assay with the serum agglutination test, the rose bengal plate test and the milk ring test for the detection of Brucella antibodies in bovine sera and milk. Zentralbl Veterinarmed B. 1999;46(4):279-85.

5. Cadmus SIAH, Stack J. The use of the milk ring test and rose bengal test in brucellosis control and eradication in Nigeria. J S Afr Vet Assoc. 2008;79(3):113-5.

6. Mathias LA, Pinto AA. Comparative study among complement fixation, serum agglutination and Rose Bengal Plate tests in the serodiagnosis of bovine brucellosis. Int J Zoonoses. 1983:10(1):1-6.

7. Uzal FA, Carrasco AE, Echaide S, Nielsen K, Robles CA. Evaluation of an indirect ELISA for the diagnosis of bovine brucellosis. J Vet Diagn Invest. 1995;7(4):473-5.

8. Nielsen K, Smith P, Gall D, Perez B, Cosma C, Mueller P, et al. Development and validation of an indirect enzyme immunoassay for detection of antibody to Brucella abortus in milk. Vet Microbiol. 1996;52(1-2):165-73.

9. Nielsen KH, Kelly L, Gall D, Nicoletti P, Kelly W. Improved competitive enzyme immunoassay for the diagnosis of bovine brucellosis. Vet Immunol Immunopathol. 1995;46(3-4):285-91.

10. Nielsen K, Smith P, Yu WL, Elmgren C, Halbert G, Nicoletti P, et al. Validation of a second generation competitive enzyme immunoassay (CELISA) for the diagnosis of brucellosis in various species of domestic animals. Vet Immunol Immunopathol. 2008;125(3-4):246-50.

11. Nielsen K, Gall D, Lin M, Massangill C, Samartino L, Perez B, et al. Diagnosis of bovine brucellosis using a homogeneous fluorescence polarization assay. Vet Immunol Immunopathol. 1998;66(3-4):321-9.

12. Saegerman C, De Waele L, Gilson D, Godfroid J, Thiange P, Michel P, et al. Evaluation of three serum i-ELISAs using monoclonal antibodies and protein $\mathrm{G}$ as peroxidase conjugate for the diagnosis of bovine brucellosis. Vet Microbiol. 2004;100(1-2):91-105.

13. Nielsen K, Smith P, Widdison J, Gall D, Kelly L, Kelly W, et al. Serological relationship between cattle exposed to Brucella abortus, Yersinia enterocolitica O:9 and Escherichia coli 0157:H7. Vet Microbiol. 2004;100(1-2):25-30.

14. Chart H, Okubadejo OA, Rowe B. The serological relationship between Escherichia coli $\mathrm{O} 157$ and Yersinia enterocolitica 09 using sera from patients with brucellosis. Epidemiol Infect. 1992;108(1):77-85.

15. Caroff M, Bundle DR, Perry MB. Structure of the O-chain of the phenol-phase soluble cellular lipopolysaccharide of Yersinia enterocolitica serotype 0:9. Eur J Biochem. 1984;139(1):195-200.

16. Nielsen K. Diagnosis of brucellosis by serology. Vet Microbiol. 2002;90(1-4):447-59.

17. Letesson JJ, Tibor A, van Eynde G, Wansard V , Weynants $V$, Denoel $P$, et al. Humoral immune responses of Brucella-infected cattle, sheep, and goats to eight purified recombinant Brucella proteins in an indirect enzyme-linked immunosorbent assay. Clin Diagn Lab Immunol. 1997;4(5):556-64.

18. Xin T, Yang H, Wang N, Wang F, Zhao P, Wang H, et al. Limitations of the BP26 protein-based indirect enzyme-linked immunosorbent assay for diagnosis of Brucellosis. Clin Vaccine Immunol. 2013;20(9):1410-7.

19. Palmer DA, Douglas JT. Analysis of Brucella lipopolysaccharide with specific and cross-reacting monoclonal antibodies. J Clin Microbiol. 1989;27(10):2331-7.

20. Weynants $\vee$, Gilson D, Cloeckaert A, Tibor A, Denoel PA, Godfroid F, et al. Characterization of smooth lipopolysaccharides and $O$ polysaccharides of Brucella species by competition binding assays with monoclonal antibodies. Infect Immun. 1997:65(5):1939-43.

21. Cloeckaert A, Weynants V, Godfroid J, Verger JM, Grayon M, Zygmunt MS O-Polysaccharide epitopic heterogeneity at the surface of Brucella spp. studied by enzyme-linked immunosorbent assay and flow cytometry. Clin Diagn Lab Immunol. 1998;5(6):862-70.
22. Alton $\mathrm{GG}$, Jones $\mathrm{LM}$, Angus RD, Verger JM. Techniques for the Brucellosis Laboratory. Institut National de la Recherche Agronomique: Paris, France; 1988.

23. Hu JQ, Li YF, Guo JQ, Shen HG, Zhang DY, Zhou JY. Production and characterization of monoclonal antibodies to (poly100)S1 protein of avian infectious bronchitis virus. Zoonoses Public Health. 2007;54(2):69-77.

24. Cardoso PG, Macedo GC, Azevedo V, Oliveira SC. Brucella spp noncanonical LPS: structure, biosynthesis, and interaction with host immune system. Microb Cell Fact. 2006;5:13.

25. Ewalt DR, Payeur JB, Rhyan JC, Geer PL. Brucella suis biovar 1 in naturally infected cattle: a bacteriological, serological, and histological study. J Vet Diagn Invest. 1997;9(4):417-20.

26. Kahler SC. Brucella melitensis infection discovered in cattle for first time, goats also infected. J Am Vet Med Assoc. 2000;216(5):648.

27. Zowghi E, Ebadi A, Mohseni B. Isolation of Brucella organisms from the milk of seronegative cows. Rev Sci Tech. 1990;9(4):1175-8.

28. O'Grady D, Byrne W, Kelleher P, O'Callaghan H, Kenny K, Heneghan T, et al. A comparative assessment of culture and serology in the diagnosis of brucellosis in dairy cattle. Vet J. 2014;199(3):370-5.

29. OIE: Manual of Diagnostic Tests and Vaccines for Terrestrial Animals. http:// www.oie.int/eng/normes/mmanual/A_summry.htm; 2009.

30. Rezania S, Amirmozaffari N, Tabarraei B, Jeddi-Tehrani M, Zarei O, Alizadeh R, et al. Extraction, Purification and Characterization of Lipopolysaccharide from Escherichia coli and Salmonella typhi. Avicenna J Med Biotechnol. 2011;3(1):3-9.

31. Coligan JE, editor. Current Protocols in Immunology. 3rd ed. Hoboken: John Wiley \& Sons,Inc; 2003.

32. Kimura M, Imaoka K, Suzuki M, Kamiyama T, Yamada A. Evaluation of a microplate agglutination test (MAT) for serological diagnosis of canine brucellosis. J Vet Med Sci. 2008;70(7):707-9.

\section{Submit your next manuscript to BioMed Central and take full advantage of:}

- Convenient online submission

- Thorough peer review

- No space constraints or color figure charges

- Immediate publication on acceptance

- Inclusion in PubMed, CAS, Scopus and Google Scholar

- Research which is freely available for redistribution 